OPEN ACCESS

Edited by:

Charles M. A. P. Franz,

Max Rubner-Institut, Germany

Reviewed by:

Daniel Cozzolino,

University of Adelaide, Australia

Giulia Tabanelli,

Centro Interdipartimentale di Ricerca

Industriale Agroalimentare, Italy

*Correspondence:

Po-Ren Hsueh

hsporen@ntu.edu.tw

Specialty section:

This article was submitted to

Food Microbiology,

a section of the journal

Frontiers in Microbiology

Received: 12 August 2015 Accepted: 26 October 2015

Published: 05 November 2015

Citation:

Lee M-R, Tsai C-J, Teng S-H, and Hsueh P-R (2015) Identification

of Weissella species by matrix-assisted laser desorption/ionization time-of-flight mass spectrometry.

Front. Microbiol. 6:1246. doi: 10.3389/fmicb.2015.01246

\section{Identification of Weissella species by matrix-assisted laser desorption/ionization time-of-flight mass spectrometry}

\author{
Meng-Rui Lee 1,2,3, Chia-Jung Tsai ${ }^{4}$, Shih-Hua Teng ${ }^{5}$ and Po-Ren Hsueh ${ }^{2,6 *}$ \\ ${ }^{1}$ Department of Internal Medicine, National Taiwan University Hospital Hsin-Chu Branch, National Taiwan University College \\ of Medicine, Hsinchu, Taiwan, ${ }^{2}$ Department of Internal Medicine, National Taiwan University Hospital, National Taiwan \\ University College of Medicine, Taipei, Taiwan, ${ }^{3}$ Institute of Epidemiology and Preventive Medicine, College of Public Health, \\ National Taiwan University, Taipei, Taiwan, ${ }^{4}$ Department of Internal Medicine, Taoyuan General Hospital, Ministry of Health \\ and Welfare, Taoyuan, Taiwan, ${ }^{5}$ Department of Graduate Institute of Biomedical Sciences, Chang Gung University, Taoyuan, \\ Taiwan, ${ }^{6}$ Department of Laboratory Medicine, National Taiwan University Hospital, National Taiwan University College of \\ Medicine, Taipei, Taiwan
}

Although some Weissella species play beneficial roles in food fermentation and in probiotic products, others such as Weissella confusa are emerging Gram-positive pathogens in immunocompromised hosts. Weissella species are difficult to identify by conventional biochemical methods and commercial automated systems and are easily misidentified as Lactobacillus and Leuconostoc species. Matrix-assisted laser desorption/ionization time-of-flight mass spectrometry (MALDI-TOF MS) is increasingly being used for bacterial identification. Little, however, is known about the effectiveness of MALDI-TOF MS in identifying clinical isolates of Weissella to the species level. In this study, we evaluated whether the MALDI-TOF MS Bruker Biotyper system could accurately identify a total of $20 \mathrm{~W}$. confusa and $2 \mathrm{~W}$. cibaria blood isolates that had been confirmed by $16 \mathrm{~s}$ rRNA sequencing analysis. The MALDI-TOF Biotyper system yielded no reliable identification results based on the current reference spectra for the two species (all score values $<1.7$ ). New $W$. confusa spectra were created by randomly selecting $3 \mathrm{~W}$. confusa isolates and external validation was performed by testing the remaining $17 \mathrm{~W}$. confusa isolates using the new spectra. The new main spectra projection (MSP) yielded reliable score values of $>2$ for all isolates with the exception of one (score value, 1.963). Our results showed that the MSPs in the current database are not sufficient for correctly identifying $W$. confusa or $W$. cibaria. Further studies including more Weissella isolates are warranted to further validate the performance of MALDI-TOF in identifying Weissella species.

\footnotetext{
Keywords: Weissella confusa, Weissella cibaria, Bacteremia, matrix-assisted laser desorption/ionization time-offlight mass spectrometry, 16S rRNA
}

\section{INTRODUCTION}

Members of Weissella species are found in various fermented food products and are considered to be potential probiotics (Nam et al., 2002; Fairfax et al., 2014; Kot et al., 2014; Fusco et al., 2015). However, some Weissella species, namely W. confusa, can cause opportunistic infections in immunocompromised hosts and have been found to be intrinsically resistant to vanomycin, 
making treatment difficult (Lee et al., 2011). W. confusa is most likely an underestimated pathogen because it shares similar staining and biochemical properties with other Gram-positive, catalase-negative bacteria such as Leuconostoc and Lactobacillus species (Facklam and Elliott, 1995; Shin et al., 2007; Schillinger et al., 2008; Lee et al., 2011; Fairfax et al., 2014).

Traditionally, correct identification of Weissella species relies on the implementation of 16S rRNA sequencing (Chelo et al., 2007; Lee et al., 2011). Matrix-assisted laser desorption/ionization time-of-flight mass spectrometry (MALDI-TOF MS) is increasingly being used to identify unusual bacterial pathogens including Weissella species isolated from clinical specimens (Lee et al., 2013; Chen et al., 2014; Fairfax et al., 2014; Hsueh et al., 2014). MALDI-TOF MS has the advantage of being faster and more cost-effective than the conventional 16S rRNA sequencing methods and is, therefore, expected to play a more important role in food and clinical laboratories (Steensels et al., 2011). Few studies, however, have addressed and tested the effectiveness of MALDI-TOF MS in identifying Weissella species.

In this study, we compared the accuracy of MALDI-TOF MS with that of $16 \mathrm{~S}$ rRNA sequencing in identifying Weissella species.

\section{MATERIALS AND METHODS}

\section{Bacterial Isolates}

A total of 147 isolates of Gram-positive rods, including Lactobacillus and Leuconostoc species, that had been recovered from patients with bloodstream infections at the National Taiwan University Hospital (a 2900-bed tertiary care center in northern Taiwan) during the period 2000-2014 were obtained from the hospital's microbiology laboratory. These isolates had initially been identified as either Lactobacillus or Leuconostoc species by traditional or commercial automated identification systems as reported previously (Lee et al., 2011, 2015). Among these isolates, 22 isolates were identified as Weisella species by colony morphology, a positive Gram stain reaction, growth at $37^{\circ} \mathrm{C}$, and negative reactions to pyrrolidonyl arylamidase and leucine aminopeptidase as well as by $16 \mathrm{~S}$ rRNA sequencing analysis.

\section{$16 S$ rRNA Gene Sequencing Analysis}

Partial sequencing of up to 1475 base pairs of the $16 \mathrm{~S}$ rRNA gene was performed for species identification of all 147 isolates of Lactobacillus and Leuconostoc species using the following primers: forward: 5' -AGAGTTTGATCCTGGCTCAG3'; reverse: 5'-GGTTACCTTGTTACGACTT-3' (Tsai et al., 2004). The results were compared with published sequences in the GenBank database using the BLASTN algorithm. The closest matches and GenBank accession numbers were obtained. L. lactis ATCC 19256 was used as the control strain in each test as described previously (Lee et al., 2011).

\section{Performance of the MALDI Biotyper}

For analysis of the 22 isolates of Weissella species by the MALDITOF Biotyper (Microflex LT; Bruker Daltonik GmbH, Bremen, Germany), the samples were prepared and analyzed as previously described (Verroken et al., 2010; Hsueh et al., 2014). Briefly, all isolates were incubated in Trypticase soy agar with 5\% sheep blood (BAP) (Becton, Dickinson Microbiology Systems, Sparks, $\mathrm{MD}$, USA) and incubated for $48 \mathrm{~h}$ at $37^{\circ} \mathrm{C}$. Colonies were transferred to a $1.5-\mathrm{ml}$ screw-cap Eppendorf tube containing $300 \mu \mathrm{l}$ of distilled water and then mixed with $900 \mu \mathrm{l}$ of ethanol by pipetting. The suspension was pelleted by centrifugation at $13,000 \mathrm{rpm}$ for $2 \mathrm{~min}$, evaporated to dryness, and then reconstituted in $50 \mu \mathrm{l}$ of $70 \%$ formic acid. After incubation for $30 \mathrm{~s}, 50 \mu \mathrm{l}$ of acetonitrile (Sigma-Aldrich) was added. The suspension was then centrifuged at 13,000 rpm for $2 \mathrm{~min}$. Next, $1.0 \mu \mathrm{l}$ of the supernatant was applied to a 96-spot polished steel target plate (Bruker Daltonik GmbH, Bremen, Germany) and dried. A saturated solution of $1.0 \mu \mathrm{l}$ of MALDI matrix ( $\alpha$-cyano-4-hydroxycinnamic acid [HCCA]; Bruker Daltonik $\mathrm{GmbH})$ was applied to each sample and dried. Measurements were performed with the Bruker microflex LT MALDI-TOF MS system (Bruker Daltonik $\mathrm{GmbH}$ ) using FlexControl software with Compass Flex Series version 1.3 software and a $60-\mathrm{Hz}$ nitrogen laser (337 $\mathrm{nm}$ wavelength). The spectra were collected in the linear positive mode in a mass range covering $\mathrm{m} / \mathrm{z} 1,960$ 20,132 . Spectra ranging from the mass-to-charge ratio $(\mathrm{m} / \mathrm{z})$ 2,000-20,000 were analyzed using Bruker Biotyper automation control and the Bruker Biotyper 3.1 software and library (database [DB] 5627 with 5,627 entries). Identification scores of $\geq 2.000$ indicated species-level identification, scores of 1.700 1.999 indicated genus-level identification, and scores of $<1.700$ indicated no reliable identification. All isolates with discrepant identification results between the molecular and Bruker Biotyper methods were retested twice.

\section{Cluster Analysis and Creation of New W. confusa and W. cibria Main Spectra Projection}

Clustering analysis of 22 isolates of the two genetically identified Weissella species collected from NTUH was performed using ClinProTools 3.0 (Bruker Daltonics GmbH, Bremen, Germany) as previously described (Chen et al., 2014; Hsueh et al., 2014). Because the current MALDI-TOF database failed to make a correct identification of all $W$. confusa and $W$. cibaria isolates, spectra of $3 W$. confusa isolates were randomly selected from the 20 isolates of $W$. confusa obtained from the NTUH for the creation of main spectra projections (MSPs; database entrance) using Bruker Biotyper MALDI-TOF MS software (Bruker Daltonics) as previously described (Chen et al., 2014; Hsueh et al., 2014). The spectra generated using the 3 isolates were blindly tested against those of the residual $17 \mathrm{~W}$. confusa isolates.

\section{RESULTS}

The MALDI-TOF Biotyper system with current reference spectra yielded no reliable identification results (score values ranged from 1.282 to 1.519 ) among the $20 \mathrm{~W}$. confusa and $2 \mathrm{~W}$. cibria isolates (Table 1). Using the spectra created by the $3 \mathrm{~W}$. confusa isolates (Isolates 1,2 , and 3 ), we further tested the residual $17 \mathrm{~W}$. confusa 
TABLE 1 | Species identification of 22 isolates of Weissella species by 16 SRNA sequencing analysis and the MALDI-TOF Biotyper system using preexisting (DB 5627) and newly established databases.

\begin{tabular}{|c|c|c|c|c|c|c|c|}
\hline \multirow[t]{2}{*}{$\begin{array}{l}\text { Isolate } \\
\text { no. }\end{array}$} & \multirow[t]{2}{*}{$\begin{array}{l}\text { Original identification } \\
\text { results by conventional } \\
\text { methods }\end{array}$} & \multicolumn{2}{|c|}{ 16S rRNA sequencing } & \multicolumn{2}{|c|}{$\begin{array}{l}\text { Identification by MALDI } \\
\text { Biotyper based on } \\
\text { database } 5627\end{array}$} & \multicolumn{2}{|c|}{$\begin{array}{l}\text { Identification by MALDI } \\
\text { Biotyper based on newly } \\
\text { developed database }\end{array}$} \\
\hline & & $\begin{array}{l}\text { Results (maximal } \\
\text { identity, \%) }\end{array}$ & Accession number & Results & Score value & $\begin{array}{c}\text { Results (best } \\
\text { match) }\end{array}$ & Score value \\
\hline $1^{*}$ & Lactobacillus species & W. confusa $(100)$ & KF245541.1 & $\mathrm{NRI}$ & 1.361 & - & - \\
\hline $2^{*}$ & Leuconostoc species & W. confusa (99.8) & EU807756.1 & $\mathrm{NRI}$ & 1.336 & - & - \\
\hline $3^{*}$ & Leuconostoc species & W. confusa $(100)$ & GU138614.1 & $N R I$ & 1.298 & - & - \\
\hline 4 & Lactobacillus species & W. confusa (100) & KJ476186.1 & $N R I$ & 1.391 & W. confusa & 2.054 \\
\hline 5 & Lactobacillus species & W. confusa (100) & KF245541.1 & $\mathrm{NRI}$ & 1.448 & W. confusa & 2.073 \\
\hline 6 & Lactobacillus species & W. confusa (100) & KF245541.1 & $\mathrm{NRI}$ & 1.29 & W. confusa & 2.250 \\
\hline 7 & Lactobacillus species & W. confusa (100) & KF245541.1 & $\mathrm{NRI}$ & 1.39 & W. confusa & 2.243 \\
\hline 8 & Lactobacillus species & W. confusa (100) & KF245541.1 & $\mathrm{NRI}$ & 1.43 & W. confusa & 2.501 \\
\hline 9 & Lactobacillus species & W. confusa (100) & KF245541.1 & $\mathrm{NRI}$ & 1.338 & W. confusa & 2.376 \\
\hline 10 & Lactobacillus species & W. confusa (100) & KJ476186.1 & $\mathrm{NRI}$ & 1.503 & W. confusa & 2.113 \\
\hline 11 & Leuconostoc species & W. confusa (99.9) & KJ476186.1 & $\mathrm{NRI}$ & 1.303 & W. confusa & 2.395 \\
\hline 12 & Leuconostoc species & W. confusa (100) & EU807756.1 & $\mathrm{NRI}$ & 1.35 & W. confusa & 2.101 \\
\hline 13 & Leuconostoc species & W. confusa (100) & EU807756.1 & $\mathrm{NRI}$ & 1.362 & W. confusa & 2.04 \\
\hline 14 & Leuconostoc species & W. confusa (99.6) & KJ476186.1 & $\mathrm{NRI}$ & 1.391 & W. confusa & 2.332 \\
\hline 15 & Lactobacillus species & W. confusa (100) & GU138614.1 & $\mathrm{NRI}$ & 1.416 & W. confusa & 2.333 \\
\hline 16 & Leuconostoc species & W. confusa (99.7) & GU138614.1 & $\mathrm{NRI}$ & 1.394 & W. confusa & 2.022 \\
\hline 17 & Leuconostoc species & W. confusa (100) & GU138614.1 & $\mathrm{NRI}$ & 1.38 & W. confusa & 1.963 \\
\hline 18 & Leuconostoc species & W. confusa (99.8) & KJ476186.1 & $\mathrm{NRI}$ & 1.282 & W. confusa & 2.368 \\
\hline 19 & Leuconostoc species & W. confusa (100) & GU138614.1 & $\mathrm{NRI}$ & 1.413 & W. confusa & 2.302 \\
\hline 20 & Lactobacillus species & W. confusa (100) & KJ476186.1 & $\mathrm{NRI}$ & 1.382 & W. confusa & 2.355 \\
\hline 21 & Leuconostoc species & W. cibaria (100) & AB510747.1 & $\mathrm{NRI}$ & 1.333 & - & - \\
\hline 22 & Leuconostoc species & W. cibaria (99.3) & AB510747.1 & $\mathrm{NRI}$ & 1.519 & - & - \\
\hline
\end{tabular}

*Isolates for creation of W. confusa spectra; NRI, no reliable identification.

isolates. We found that all of the $W$. confusa isolates with the exception of one (Isolate 17, score value of 1.963) were identified correctly with a score $>2$ using the three newly created MSPs. The highest scores were achieved with MSP created by W. confusa Isolate 1 in the ten $W$. confusa isolates, MSP created by $W$. confusa Isolate 2 in the five $W$. confusa isolates and MSP created by $W$. confusa Isolate 3 in the W. confusa isolate.

The characteristic spectra of $W$. confusa (Isolate 8) and W. cibria (Isolate 22) are illustrated in Figure 1. The characteristic peaks for $W$. cibria were 3008.1, 3634.1, 4079.8, 4954.5, 5380.2, 6371.5, 7267.7, 7962.6, 9530.7, and 11028.2. The characteristic peaks for $W$. confusa were $3010.1,3585.2,4162.9,4608.2,4844.0$, 5183.7, 6404.8, 7167.4, 7964.3, 9215.7, and 9686.8.

\section{DISCUSSION}

We found that current reference spectra in the MALDI-TOF database were much less accurate than 16s rRNA sequencing in correctly identifying $W$. confusa and $W$. cibaria. Using the $W$. confusa MSPs created by our database, we were able to correctly identify all $W$. confusa isolates.

It is notoriously difficult to identify $W$. confusa. The $16 \mathrm{~S}$ rRNA sequencing method is considered the standard identification method, although other methods have shown to be able to accurately identify $W$. confusa. A novel PCRbased identification method using amplified fragment length polymorphism (AFLP)-derived marker was shown to have $100 \%$ specificity in identifying W. confusa (Fusco et al., 2011).

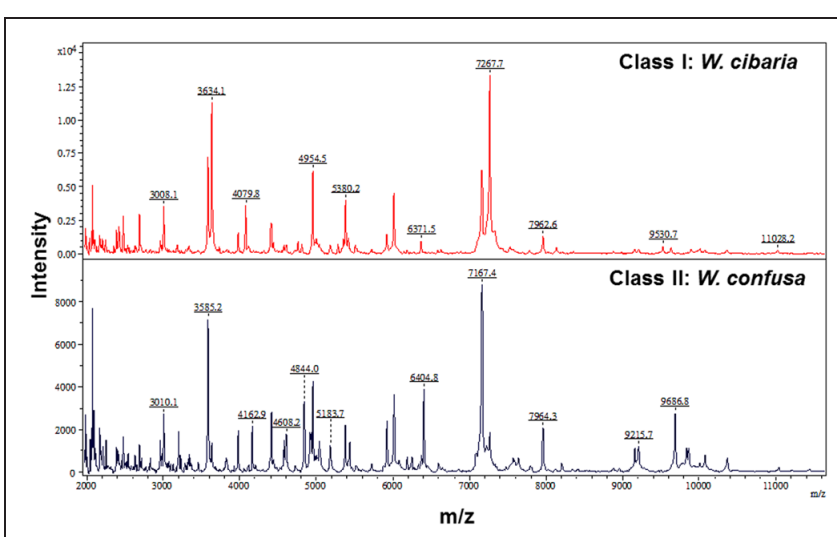

FIGURE 1 | Two clusters of Weissella species spectra, i.e., cluster I (W. cibaria) and cluster II (W. confusa), analyzed by clustering analysis of MALDI-TOF MS results. The absolute intensities of the ions are shown on the $y$ axis and the masses $(\mathrm{m} / \mathrm{z})$ of the ions are shown on the $x$ axis. The $\mathrm{m} / \mathrm{z}$ values represent the mass-to-charge ratio. 
MALDI-TOF has also been shown to correctly identify the organism in blood samples (Lee et al., 2013; Fairfax et al., 2014). Despite satisfying results, it has to be kept in mind that the tested case numbers were small and only five Weissella species are listed in the MALDI-TOF database, namely $W$. confusa, $W$. halotolerans, W. kandleri, W. minor, and $W$. viridescens. Stevenson et al. (2010) reported that only one W. confusa isolate achieved a score value of 1.7 , indicating an identification only at genus level. In our study, all of the $W$. confusa isolates that had been correctly identified by $16 \mathrm{~S}$ rRNA sequencing failed to be identified with MALDI-TOF using the current database. The discrepancy between the two identification methods remains to be solved. Further studies comparing the results between $16 \mathrm{~S}$ rRNA sequencing and MALDI-TOF are warranted.

The most well-studied food product in association with Weissella species is kimchi, a traditional Korean food. Lactic acid bacteria, belonging to the genera Leuconostoc, Lactobacillus, and Weissella, are considered to be the most likely organisms responsible for kimchi fermentation (Lee et al., 2005; Jung et al., 2013; Kwak et al., 2014), and studies have shown that $W$. confusa is involved in the early and middle stages of kimchi fermentation (Lee et al., 2005; Kim et al., 2012). $W$. confusa has also been detected in food products from other countries, such as fermented milk products in Ghana, sourdough products in Italy, fermented soy sauce in Taiwan, and in soy sauce brine in Malaysia (Corsetti et al., 2001; Akabanda et al., 2013; Wei et al., 2013; Sulaiman et al., 2014).

Although $W$. ceti is well known in veterinary medicine for causing weissellosis in rainbow trout (Snyder et al., 2015), to the best of our knowledge, only $W$. confusa has been linked to human invasive infections. Therefore, other Weissella species are probably safer alternatives to $W$. confusa as probiotics.

Bacteremia due to $W$. confusa is rare and tends to occur in severely immunocompromised hosts, such as patients who have received organ transplants (Harlan et al., 2011; Lee et al., 2011, 2013; Salimnia et al., 2011; Fairfax et al., 2014). Immunocompromised hosts undergoing abdominal surgery have also been shown to be at particular risk since abdominal surgery results in the breakdown of intestinal mucosa (Lee et al., 2011). Given the findings of our study, the incidence of bacteremia due

\section{REFERENCES}

Akabanda, F., Owusu-Kwarteng, J., Tano-Debrah, K., Glover, R. L., Nielsen, D. S., and Jespersen, L. (2013). Taxonomic and molecular characterization of lactic acid bacteria and yeasts in nunu, a Ghanaian fermented milk product. Food Microbiol. 34, 277-283. doi: 10.1016/j.fm.2012.09.025

Chelo, I. M., Zé-Zé, L., and Tenreiro, R. (2007). Congruence of evolutionary relationships inside the Leuconostoc-Oenococcus-Weissella clade assessed by phylogenetic analysis of the $16 \mathrm{~S}$ rRNA gene, dnaA, gyrB, rpoC and dnaK. Int. J. Syst. Evol. Microbiol. 57(Pt 2), 276-286. doi: 10.1099/ijs.0.64468-0

Chen, P. L., Lee, T. F., Wu, C. J., Teng, S. H., Teng, L. J., Ko, W. C., et al. (2014). Matrix-assisted laser desorption ionization-time of flight mass spectrometry can accurately differentiate Aeromonas dhakensis from A. hydrophila, A. caviae, and A. veronii. J. Clin. Microbiol. 52, 2625-2628. doi: 10.1128/JCM.0 1025-14 to $W$. confusa is underestimated because of the difficulties in making a correct identification with commercial and biochemical methods. The findings also suggest that immunocompromised patients should refrain from consuming fermented foods.

In our previous study, the results of $16 \mathrm{~S}$ rRNA sequencing analysis showed that 10 of 43 blood isolates of catalase-negative, vancomycin-resistant coccobacilli were actually $W$. confusa (Lee et al., 2011). In this study, all Weissella species were either identified as Lactobacillus or Leuconostoc species by commercial automatic systems or traditional biochemical methods. This finding is in concordance with a phylogenetical study by Chelo et al. (2007), who showed that Leuconostoc species and Lactobacillus species were close to $W$. confusa in neighbor-joining tree by $16 \mathrm{~S}$ rRNA sequencing. The applications of $16 \mathrm{~S}$ rRNA sequencing may lead to a better understanding of the incidence of bacteremia due to Weissella species.

During the study period, the actual number of bacteremia due to Weissella species might be under-estimated. In this study, we did not perform 16S rRNA sequencing on bacteremic isolates of Gram-positive cocci, including viridians group streptococci, Pediococcus, and Gemella species. Some Weissella species were misidentified as these organisms by conventional identification methods (Ruoff, 2002; Lee et al., 2011). In this study, 16S rRNA sequencing was only performed on bacteremic isolates of Lactobacillus and Leuconostoc species, which were the most closely relevant species and thus were considered the most possible bacteria $W$. confusa could be misidentified as (Chelo et al., 2007; Lee et al., 2011).

\section{CONCLUSION}

The MALDI Biotyper system is less accurate that 16S rRNA sequencing in correctly identifying Weissella species. W. confusa is an underestimated cause of bacteremia due to its tendency to be misidentified. More extensive epidemiologic studies with molecular methods are needed to reveal the true prevalence and incidence of invasive $W$. confusa infections. Also, studies involving more clinical Weissella isolates are needed to explore the effectiveness of the MALDI Biotyper system in identification of Weissella species.

Corsetti, A., Lavermicocca, P., Morea, M., Baruzzi, F., Tosti, N., and Gobbetti, M. (2001). Phenotypic and molecular identification and clustering of lactic acid bacteria and yeasts from wheat (species Triticum durum and Triticum aestivum) sourdoughs of Southern Italy. Int. J. Food Microbiol. 64, 95-104. doi: 10.1016/S0168-1605(00)00447-5

Facklam, R., and Elliott, J. A. (1995). Identification, classification, and clinical relevance of catalase-negative, gram-positive cocci, excluding the streptococci and enterococci. Clin. Microbiol. Rev. 8, 479-495.

Fairfax, M. R., Lephart, P. R., and Salimnia, H. (2014). Weissella confusa: problems with identification of an opportunistic pathogen that has been found in fermented foods and proposed as a probiotic. Front. Microbiol. 5:254. doi: 10.3389/fmicb.2014.00254

Fusco, V., Quero, G. M., Cho, G. S., Kabisch, J., Meske, D., Neve, H., et al. (2015). The genus Weissella: taxonomy, ecology and biotechnological potential. Front. Microbiol. 6:155. doi: 10.3389/fmicb.2015.00155 
Fusco, V., Quero, G. M., Stea, G., Morea, M., and Visconti, A. (2011). Novel PCR-based identification of Weissella confusa using an AFLP-derived marker. Int. J. Food Microbiol. 145, 437-443. doi: 10.1016/j.ijfoodmicro.201 1.01 .015

Harlan, N. P., Kempker, R. R., Parekh, S. M., Burd, E. M., and Kuhar, D. T. (2011). Weissella confusa bacteremia in a liver transplant patient with hepatic artery thrombosis. Transpl. Infect. Dis. 13, 290-293. doi: 10.1111/j.13993062.2010.00579.x

Hsueh, P. R., Lee, T. F., Du, S. H., Teng, S. H., Liao, C. H., Sheng, W. H., et al. (2014). Bruker biotyper matrix-assisted laser desorption ionization-time of flight mass spectrometry system for identification of Nocardia, Rhodococcus, Kocuria, Gordonia, Tsukamurella, and Listeria species. J. Clin. Microbiol. 52, 2371-2379. doi: 10.1128/JCM.00456-14

Jung, J. Y., Lee, S. H., Jin, H. M., Hahn, Y., Madsen, E. L., and Jeon, C. O. (2013). Metatranscriptomic analysis of lactic acid bacterial gene expression during kimchi fermentation. Int. J. Food Microbiol. 163, 171-179. doi: 10.1016/j.ijfoodmicro.2013.02.022

Kim, B., Seo, W. T., Kim, M. G., Yun, H. D., and Cho, K. M. (2012). Metagenomic lactic acid bacterial diversity during Mulkimchi fermentation based on 16S rRNA sequence. J. Korean Soc. Appl. Biol. Chem. 55, 787-792. doi: 10.1007/s13765-012-2185-3

Kot, W., Neve, H., Heller, K. J., and Vogensen, F. K. (2014). Bacteriophages of Leuconostoc, Oenococcus, and Weissella. Front. Microbiol. 5:186. doi: 10.3389/fmicb.2014.00186

Kwak, S. H., Cho, Y. M., Noh, G. M., and Om, A. S. (2014). Cancer preventive potential of kimchi lactic acid bacteria (Weissella cibaria, Lactobacillus plantarum). J. Cancer Prev. 19, 253-258. doi: 10.15430/JCP.2014.19.4.253

Lee, J. S., Heo, G. Y., Lee, J. W., Oh, Y. J., Park, J. A., Park, Y. H., et al. (2005). Analysis of kimchi microflora using denaturing gradient gel electrophoresis. Int. J. Food Microbiol. 102, 143-150. doi: 10.1016/j.ifoodmicro.2004.12.010

Lee, M. R., Huang, Y. T., Liao, C. H., Lai, C. C., Lee, P. I., and Hsueh, P. R. (2011). Bacteraemia caused by Weissella confusa at a university hospital in Taiwan, 1997-2007. Clin. Microbiol. Infect. 17, 1226-1231. doi: 10.1111/j.14690691.2010.03388.x

Lee, M. R., Tsai, C. J., Liang, S. K., Lin, C. K., Huang, Y. T., and Hsueh, P. R. (2015). Clinical characteristics of bacteraemia caused by Lactobacillus spp. and antimicrobial susceptibilities of the isolates at a medical centre in Taiwan, 2000-2014. Int. J. Antimicrob. Agents 46, 439-445. doi: 10.1016/j.ijantimicag.2015.06.017

Lee, W., Cho, S. M., Kim, M., Ko, Y. G., Yong, D., and Lee, K. (2013). Weissella confusa bacteremia in an immune-competent patient with underlying intramural hematomas of the aorta. Ann. Lab. Med. 33, 459-462. doi: 10.3343/alm.2013.33.6.459

Nam, H., Ha, M., Bae, O., and Lee, Y. (2002). Effect of Weissella confusa strain PL9001 on the adherence and growth of Helicobacter pylori. Appl. Environ. Microbiol. 68, 4642-4645. doi: 10.1128/AEM.68.9.4642-4645.2002

Ruoff, K. L. (2002). Miscellaneous catalase-negative, gram-positive cocci: emerging opportunists. J. Clin. Microbiol. 40, 1129-1133. doi: 10.1128/JCM.40.4.11291133.2002

Salimnia, H., Alangaden, G. J., Bharadwaj, R., Painter, T. M., Chandrasekar, P. H., and Fairfax, M. R. (2011). Weissella confusa: an unexpected cause of vancomycin-resistant gram-positive bacteremia in immunocompromised hosts. Transpl. Infect. Dis. 13, 294-298. doi: 10.1111/j.1399-3062.2010.0 0586.x

Schillinger, U., Boehringer, B., Wallbaum, S., Caroline, L., Gonfa, A., Huch Née Kostinek, M., et al. (2008). A genus-specific PCR method for differentiation between Leuconostoc and Weissella and its application in identification of heterofermentative lactic acid bacteria from coffee fermentation. FEMS Microbiol. Lett. 286, 222-226. doi: 10.1111/j.1574-6968.2008.01286.x

Shin, J. H., Kim, D. I., Kim, H. R., Kim, D. S., Kook, J. K., and Lee, J. N. (2007). Severe infective endocarditis of native valves caused by Weissella confusa detected incidentally on echocardiography. J. Infect. 54, e149-e151. doi: 10.1016/j.jinf.2006.09.009

Snyder, A. K., Hinshaw, J. M., and Welch, T. J. (2015). Diagnostic tools for rapid detection and quantification of Weissella ceti NC36 infections in rainbow trout. Lett. Appl. Microbiol. 60, 103-110. doi: 10.1111/lam.12365

Steensels, D., Verhaegen, J., and Lagrou, K. (2011). Matrix assisted laser desorption ionization time of flight mass spectrometry for the identification of bacteria and yeast in clinical microbiological laboratory: a review. Acta Clin. Belg. 66, 267-273.

Stevenson, L. G., Drake, S. K., and Murray, P. R. (2010). Rapid identification of bacteria in positive blood culture broths by matrix-assisted laser desorption ionization-time of flight mass spectrometry. J. Clin. Microbiol. 48, 444-447. doi: 10.1128/JCM.01541-09

Sulaiman, J., Gan, H. M., Yin, W. F., and Chan, K. G. (2014). Microbial succession and the functional potential during the fermentation of chinese soy sauce brine. Front. Microbiol. 5:556. doi: 10.3389/fmicb.2014.00556

Tsai, J. C., Teng, L. J., and Hsueh, P. R. (2004). Direct detection of bacterial pathogens in brain abscesses by polymerase chain reaction amplification and sequencing of partial 16S ribosomal deoxyribonucleic acid fragments. Neurosurgery 55, 1154-1162. doi: 10.1227/01.NEU.0000140842.3 7422.EE

Verroken, A., Janssens, M., Berhin, C., Bogaerts, P., Huang, T. D., Wauters, G., et al. (2010). Evaluation of matrix-assisted laser desorption ionization-time of flight mass spectrometry for identification of Nocardia species. J. Clin. Microbiol. 48, 4015-4021. doi: 10.1128/JCM.01234-10

Wei, C. L., Chao, S. H., Tsai, W. B., Lee, P. S., Tsau, N. H., Chen, J. S., et al. (2013). Analysis of bacterial diversity during the fermentation of inyu, a hightemperature fermented soy sauce, using nested PCR-denaturing gradient gel electrophoresis and the plate count method. Food Microbiol. 33, 252-261. doi: 10.1016/j.fm.2012.10.001

Conflict of Interest Statement: The authors declare that the research was conducted in the absence of any commercial or financial relationships that could be construed as a potential conflict of interest.

Copyright (c) 2015 Lee, Tsai, Teng and Hsueh. This is an open-access article distributed under the terms of the Creative Commons Attribution License (CC BY). The use, distribution or reproduction in other forums is permitted, provided the original author(s) or licensor are credited and that the original publication in this journal is cited, in accordance with accepted academic practice. No use, distribution or reproduction is permitted which does not comply with these terms. 